species. The US Agency for International Development plans a two-year survey of animals ranging from rodents to livestock to domestic dogs and cats. These animals may not be natural reservoirs of Ebola, but they could contribute to spillovers into humans, says Dennis Carroll, director of the agency's Pandemic Influenza and Other Emerging Threats Unit.

But with so many question marks hovering over the identity of Ebola's reservoirs, some scientists say that it is time to eschew virus hunting in specific creatures and instead pursue moreholistic approaches that examine ecological and anthropological factors common to spillovers.

Tony Goldberg, an epidemiologist at the University of Wisconsin-Madison, is one such advocate. He no longer subscribes to the view that "we have to blanket the continent of Africa with field-deployable DNA sequencers and sample everything that crawls, flies or swims and eventually we'll come across it. I used to think that way," he says, "but I'm cooling off to that approach."

His team is studying how bush-meat hunters interact with wild ecosystems to identify factors that might be linked to the spillover of zoonotic infections such as Ebola.

In a similar effort, a team led by Pigott and his colleague epidemiologist Simon Hay is looking at past outbreaks for common ecological factors, such as vegetation, elevation and the presence of suspected reservoir species such as fruit bats and carriers such as apes. By modelling these data, the team has created a map of areas at risk of Ebola spillovers ${ }^{3}$.

And Barbara Han, a disease ecologist at the
Cary Institute of Ecosystem Studies in Millbrook, New York, is using machine-learning techniques to predict which bat species are likely to harbour Ebola and related viruses because they share ecological factors common to suspected reservoir species.

Research on Ebola therapies and vaccines saw an infusion of public and private funding during the epidemic, and scientists hunting the virus in the wild hope to capture the same sense of urgency and financial support. But they know that the job won't be easy. "It has lit a fire under people's butts, mine included," says Goldberg. "The problem is, we're not sure what to do with the fire."

1. Leroy, E. M. et al. Nature 438, 575-576 (2005).

2. Saéz, A. M. et al. EMBO Mol. Med. 7, 17-23 (2014).

3. Piggott, D. M. et al. eLife 3, e04395 (2014).

\title{
Rebooted Kepler spacecraft hauls in the planets
}

\section{Worlds found by K2 mission push beyond original discoveries.}

\section{BY ALEXANDRA WITZE, KISSIMMEE, FLORIDA}

$\mathrm{I}$ $\mathrm{n}$ the second phase of its life as a planet hunter, NASA's Kepler spacecraft is raking in exoplanet discoveries that are surprisingly different from those found during its first iteration.

Between 2009 and 2013, Kepler became the most successful planet-hunting machine ever, discovering at least 1,030 planets and more than 4,600 possible others in a single patch of sky. When a mechanical failure stripped the spacecraft of its ability to point precisely among the stars, engineers reinvented it in 2014 as the K2 mission, which looks at different parts of the cosmos for shorter periods of time.

In its first year of observing, $\mathrm{K} 2$ has netted more than 100 confirmed exoplanets, says astronomer Ian Crossfield at the University of Arizona in Tucson. They include a surprising number of systems in which more than one planet orbits the same star (E. Sinukoff et al. Preprint at http://arxiv.org/abs/1511.09213; 2015). The K 2 planets are also orbiting hotter stars than are many of the Kepler discoveries.

"This is really showing the power and potential of K2," says Crossfield. “These are things we never found with four years of Kepler data." He and other scientists reported the findings last week at a meeting of the American Astronomical Society in Kissimmee, Florida.
The original Kepler mission was designed to answer a specific question: what fraction of Sun-like stars have Earth-sized planets around them? Unbound by those constraints - even if not as good at pointing itself - K2 has been able to explore wider questions of planetary origin and evolution. "Now we get to look at a much bigger variety," says Steve Howell, the mission's project scientist at NASA's Ames Research Center in Moffett Field, California.

And because K2 looks at stars that are generally brighter and closer to Earth than Kepler did, the exoplanets that the mission finds are likely to be the best-studied for the foreseeable future. This is because they are near enough to allow astronomers to explore them with other telescopes on Earth and in space.

\section{UNEXPECTED BOUNTY}

In the past year, K2 has uncovered not just planets - such as three super-Earths orbiting a single star - but also surprises such as the disintegrating remains of a planet swirling around a white dwarf star. It has even probed exploding stars - because K2 stares constantly at a patch of the sky, it is able to catch a supernova as it brightens instead of later in its explosion, as other telescopes typically do.

Among the K2 planets confirmed so far, 58 are singletons, 28 come from systems with at least 2 planets and 14 are triples, Crossfield says. In addition, K2 has unearthed more than 200 candidate planets, says Andrew
Vanderburg, an astronomer at the Harvard Smithsonian Center for Astrophysics in Cambridge, Massachusetts.

K2 observes a larger fraction of the cool stars known as M dwarfs - the most common type of star in the Galaxy - than Kepler did. But surprisingly, fewer of the K2 planets are orbiting M-dwarf stars. A higher percentage of them, at least so far, circle stars that are hotter and more like the Sun, says Courtney Dressing, an astronomer at the California Institute of Technology (Caltech) in Pasadena.

K2 will begin a new type of planet-hunting on 7 April. Normally the spacecraft searches for a temporary dimming of a star caused when a planet crosses in front of it. For just under three months, however, it will look for the temporary brightening of cosmic objects, such as a galaxy, caused when a planet bends light as it crosses the line of sight between the object and the observer. The team expects to catch between 85 and 120 of these 'microlensing' planets during the campaign.

The survey will involve other telescopes and be the first automated search to be done simultaneously from the ground and in space, says Calen Henderson, an astronomer at NASA's Jet Propulsion Laboratory in Pasadena, California.

That means much more work ahead for mission scientists. "Kepler was one field and it ruined your summer," says Caltech astronomer David Ciardi. "K2 is ruining our whole year." 